\title{
Leakage Assessment of Pressure-Exchange Wave Rotors
}

\author{
P. Akbari* and M. R. Nalim ${ }^{\dagger}$ \\ Indiana University-Purdue University at Indianapolis, Indianapolis, Indiana 46202 \\ and \\ E. S. Donovan ${ }^{\ddagger}$ and P. H. Snyder ${ }^{\S}$ \\ Rolls-Royce North American Technologies, Inc._LibertyWorks, Indianapolis, Indiana 46241
}

DOI: $10.2514 / 1.31725$

\begin{abstract}
Control of leakage within wave rotors is recognized as a key requirement for efficient operation. Previous studies suggest that experimentally observed performance degradation is substantially due to flow leakage. This work presents a leakage model for predicting leakage-attributed performance degradation of four-port pressureexchange wave rotors more accurately than previous single-cavity leakage models. The methodology comprehensively considers the leakage paths of the entire device. It combines a wave-rotor quasi-one-dimensional computational fluid dynamics prediction code, experimentally validated for internal gas dynamics, with the generalized flow-circuit-modeling capability of a leakage-flow network solver. The computational fluid dynamics program and the network solver step through a series of iterations by sharing common leakage information. Application to a well-instrumented wave-rotor rig is briefly summarized, providing important guidance for the improvement of leakage and performance. The new approach is anticipated to be useful in enhancing the design, operation, and efficiency of a broad class of wave rotors by better understanding leakage and hence designing sealing features to control leakage flows.
\end{abstract}

\section{Introduction}

$\mathbf{F}$ URTHER performance improvement of aircraft engines based on conventional Brayton-cycle gas turbines is stymied by impassable material and component limits. The development of advanced thermodynamic cycles employing unsteady-flow devices (e.g., a wave-rotor machine) is foreseen as a potentially effective way forward that circumvents these limits. A wave rotor uses compression and expansion processes through unsteady waves to transfer energy between fluids in its channels. Many applications of wave rotors have been developed since their inception in the early 1940s [1]. Examples include piston-engine supercharging [2], windtunnel test facilities [3], equalizing or dividing pressure between gas streams [4]. refrigeration cycles [5], gas-turbine topping cycles [6], and pressure-gain combustion systems [7]. Details of wave-rotor operation and applications are reported in the literature $[8,9]$.

The common feature of all wave rotors is an array of channels arranged around the axis of a rotating drum, as schematically shown in Fig. 1. Through rotation, the channel ends are periodically connected to ports located on stationary end plates. The port locations and conditions are designed to generate gas-dynamic compression and expansion waves that effect pressure and energy exchange between fluids dynamically (i.e., without pressure equilibration).

As lopping units in gas-turbine engines, pressure-exchange wave rotors promise higher cycle pressure ratios and higher cycle peak temperatures without increasing turbine inlet temperature. In a particular wave-rotor-enhanced cycle of present interest (Fig. 2), air received from the compressor through a low-pressure-air inlet duct

Presented as Paper 4719 at the 42nd AIAA/ASME/SAE/ASEE Joint Propulsion Conference and Exhibit, Sacramento, CA, 9-12 July 2006; received 20 April 2007 ; accepted for publication 28 January 2008 . Copyright (1) 2008 by the American Institute of Aeronautics and Astronautics, Inc. All rights reserved. Copies of this paper may be made for personal or internal use, on condition that the copier pay the $\$ 10.00$ per-copy fee to the Copyright Clearance Center, Inc., 222 Rosewood Drive, Danvers, MA 01923; include the code $0748-4658 / 08 \$ 10.00$ in correspondence with the CCC.

*Postdoctoral Fellow, Department of Mechanical Engineering; currently Assistant Professor, Manhattan College, NY, 10471. Member AIAA.

Associate Professor, Department of Mechanical Engineering. Associate Fellow AIAA (Corresponding Author).

Senior Engineer, Heat Transfer.

Consultant Engineer, Propulsion System Integration. Member AIAA. (state 1) is compressed further by shock waves in the rotor and discharged to the combustion chamber through the high-pressure exit duct (state 2). The burned gas reenters the wave rotor through the high-pressure-gas inlet duct (state 3 ), generating a shock wave that compresses the low-pressure air. The burned gas then undergoes expansion waves and is delivered to the downstream turbine through the low-pressure-gas exit duct (state 4). The turbine inlet pressure is typically 15 to $20 \%$ higher than compressor discharge pressure [10]. a net performance benefit that may be compared with other pressuregain combustion systems, including pulse combustors [11], pulse detonation engines [12], and internal-combustion wave rotors [13].

\section{Technical Challenges: Leakage and Sealing Issues}

Despite the attractive aerodynamic and mechanical features of wave rotors, technical challenges that impede commercialization include flow leakage, localized thermal and mechanical loads, rotor blade fatigue, ducting, and limitations of design and analysis tools [9]. Most of these issues are due to the inherent flow unsteadiness (that is also the key to its usefulness) and the integration of the waverotor unconventional geometry with conventional turbomachinery components. In particular, leakage flow through the clearance gap between the rotor channels and stationary endwalls is recognized is a significant spoiler of the expected pressure gain $[9,14]$. In traditiond approaches to limiting leakage, the gap between the end plates and the rotor is minimized, but without rubbing contact, under all operating and thermal expansion conditions. The leakage flow is primarily radial, with a strong circumferential variation, in contrastio the axisymmetric and usually axial direction of leakages in a gas turbine. This is inherent to the wave rotor, in that strong pressure variation exists in the circumferential direction. Further, the clearance gap can vary due to both thermal distortions caused by nonuniform rotor or endwall heating and deflections caused by the skewed pressure loading. Attempts to minimize gap leakage by using a movable endwall may create unintended leakage paths behind to endwall. It is therefore important to understand the sensitiving of wave-rotor performance to details of the leakage process, as well is to other design and operating parameters.

Figure 3 schematically illustrates [14] leakage between a rot channel and endwalls to the wave-rotor casing. Various sell strategies such as brush seals [15] have been employed to mitizat leakage while accommodating thermal growth. Few computabioal methods have been developed to model and predict the performate 


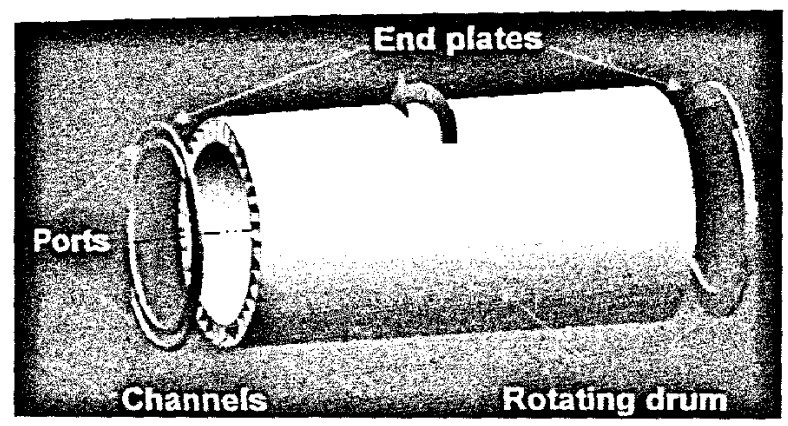

Fig. I Schematic exploded view of a typical wave rotor.

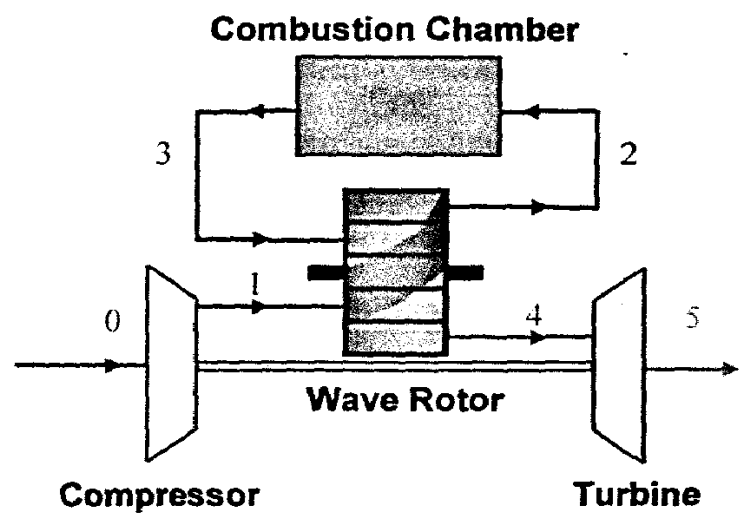

Fig. 2 Wave-rotor topping cycle.

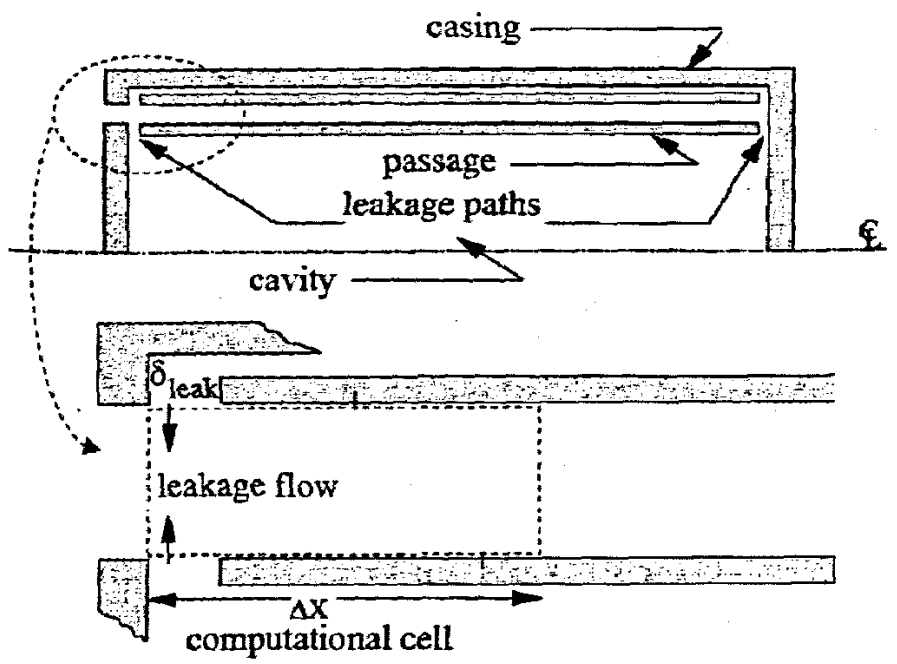

Fig. 3 Leakage flow between channels and endwalls, from [14].

degradation due to leakage flow. The single-cavity model [14] has been repeatedly employed in recent wave-rotor numerical simulations. This model is used in a quasi-one-dimensional (Q1D) computation code for flow analysis of pressure exchangers [16] and combustion wave rotors [17]. The code simulates a representative channel as it passes over various ports. In the single-cavity model, it is assumed that leakage flow is exchanged between the channels and a common cavity surrounding the rotor and the ports, driven by the pressure difference between the channel end and the cavity. The cavity state is considered constant while computing one gas-dynamic operating cycle and is updated after each cycle, assuming the timescale of cavity transients is much longer than the wave cycle time. The isentropic-nozzle-flow equation with an assumed discharge coefficient is used to calculate the leakage flow at each end of the channel. Leakage from channel to channel is neglected. The effect of leakage appears as source terms in the governing continuity and energy equations.
Although the described single-cavity model was an important step toward estimating the performance degradation due to leakage, the model is not adequate to either capture the complexity of seal leakage phenomenon or to provide sufficient guidance for improved seal design. For example, the model does not take into account any variation in cavity pressure, cavity temperature, type of sealing, or clearance gap in the circumferential direction, nor between the two ends of the rotor and between the inner and outer radii. The parameters used to calculate the leakage flow are not discemible using only geometric information and thus must be experimentally validated. For instance, the discharge coefficient in the nozzle-flow equation is a constant but arbitrary parameter, resulting in leakage estimates that may not reflect local physics even if globally calibrated. Therefore, for a more accurate wave-rotor analysis, a more comprehensive leakage-prediction model is required.

\section{Segmented Boundary Leakage Model}

A new leakage-modeling approach is developed to calculate local gap flow rates of the wave rotor. The leakage paths within the casing are represented in part by multiple stationary leakage-chamber segments located on each outer and inner radius of the channel ends in which the working fluid can radially enter or exit them, as schematically shown in Fig. 4 for one end. The other end of the rotor, not shown, may have a different number of leakage chambers. In contrast to the previous single-cavity modeling used in Q1D, the new model takes into account the variations in pressures and temperatures determining gas exchange at the local leakage sites circumferentially around the wave rotor. When combined with a flow network solver (as described in Sec. IV) of the type typically used to predict cooling and leakage flows within gas turbines, the new approach provides useful insight to guide control of leakage flows and in the design of improved wave-rotor-based engines.

An unwrapped, or developed, view of a wave rotor is illustrated in Fig. 5 with leakage gaps in communication with multiple leakage regions or chamber segments arranged around the outer and inner radii of the rotor ends. The vertical direction represents the timevarying circumferential location of the channels. The dark vertical lines represent the endwall-closed phases of the cycle, and the open ports establish the phases in which bulk flow may enter or leave the rotor channels. The boundaries of left ports $\theta_{L}$, right ports $\theta_{R}$, and leakage segments at the left (SL) and right (SR) are numbered sequentially according to the general-purpose terminology of the Q1D computational code used in this study. In this illustration, thete

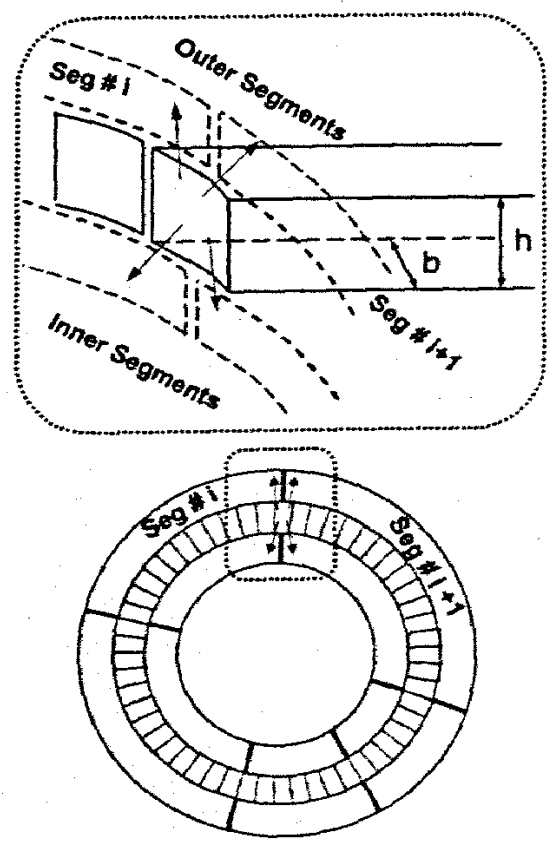

Fig. 4 Schematic of leakage phenomenon from rotating chanr stationary leakage-chamber segments. 


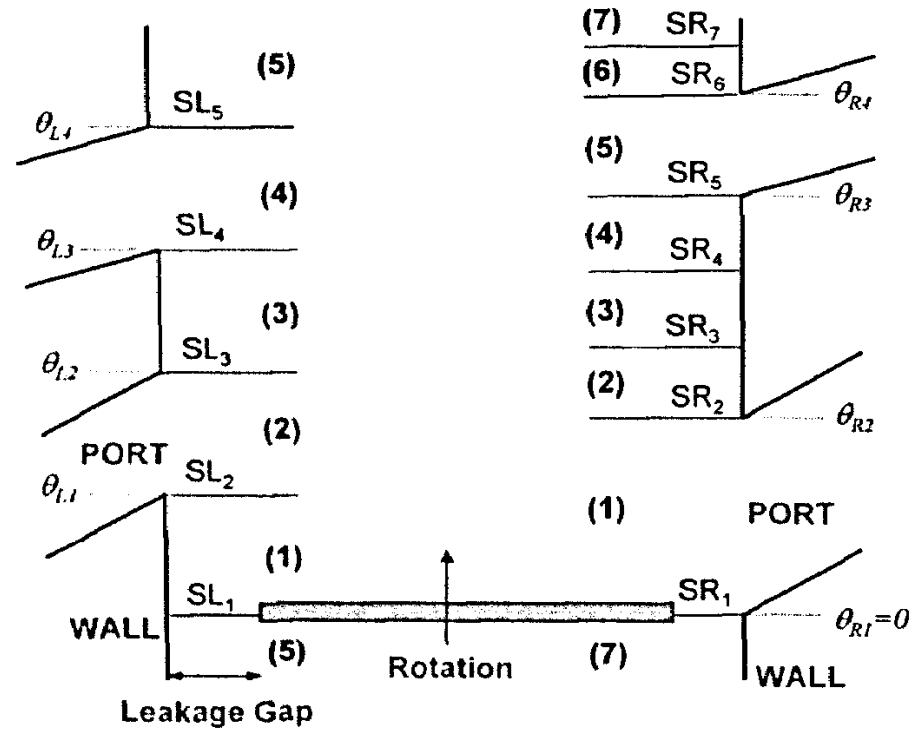

Fig. 5 Developed view of the rotor, indicating port and leakagechamber locations.

are two ports at each end and multiple leakage segments are shown for one radius. Segment boundaries are generally chosen based on the location of ports and regions in which significant changes occur in channel pressure and temperature due to the internal movement of waves and gas interfaces. On this basis, the outer and inner gaps at a given end are assigned the same segment boundaries, but this may be generalized in the future if warranted. Proper attention to the selection of the segment boundaries was required so that within a particular circumferential segment, the flow properties and leakage flow rate per unit length would be relatively uniform. The accuracy can be improved by the selection of a greater number of segments. The QID analysis is applied to a representative channel, but mass flow rates for the whole rotor are calculated by taking into account the number of channels.

The selection of segments based on identifying regions of relatively similar pressure and temperature around the circumference captures the essential feature of wave rotors that drives leakage: circumferential pressure variation. The direction and rate of local leakage between each region and the adjacent rotor channel is determined by the local pressure difference and upstream gas density. With typical good practice in casing and seal design approaches, the leakage flow is moderately well confined within each circumferential region and will not readily mix with adjacent regions except by cincumferential bulk flow driven by both pressure differences and windage. Thus. the temperature of the leakage gas within each of the regions around the circumference is well characterized by a bulk temperature in that region. The linkage of the segments in the circumferential (as well as axial) directions by bulk convection is described later. The choice of segment boundaries allows the QID code to avoid directly modeling multiple channels, because leakage between channels within a segment will be relatively small. This model is specifically relevant to the wave-rotor rig addressed here and introduces flow physics that would be present in all wave-rotor configurations.

At any instant, each end of the channel may encounter a single pair of leakage chambers (inner and outer) or it may be positioned between two sequential chamber pairs (partial opening and closing). Schematically. Fig. 6 shows one channel in the gradual opening/ closing transition between two arbitrary segments $i$ and $i-1$ at the left side, whereas the right side of the channel encounters only segment $j$ at the same instant.

In more detail, Fig. 7 represents the specific case when the moving channel of total width $\theta_{\mathrm{OT}}$ is leaving segment 5 and entering segment $l$ at the left side of the rotor. Subscripts $A$ and $B$ are used to represent the portion of the channel width exposed to the segment chambers that are ahead of and behind the channel, respectively. Here, $\theta_{A}$ and $\theta_{B}$ are exposed arc lengths to the front segment 1 and

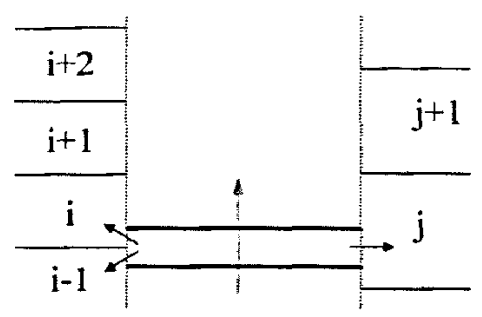

Fig. 6 Position of a chamnel relative to leakage segments, gradual opening/closing.

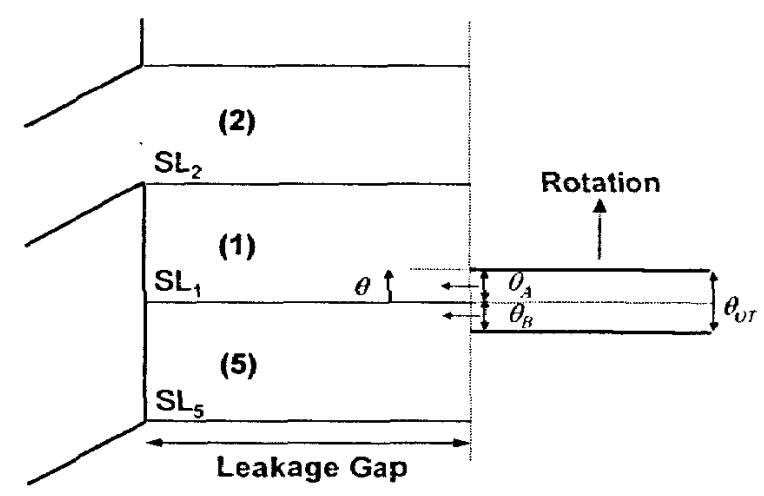

Fig. 7 Schematic model for partial opening/closing.

rear segment 5 , respectively, and $\theta$ represents the distance between the leading or forward edge of the channel and a reference angular position $\left(\theta_{R 1}=0\right.$ in Fig. 5) at any time step.

The primary objective of a leakage model is to predict leakage mass flow rates between the rotating channel and stationan segments. Consistent with QID convention, a positive mass flow rate is obtained when the flow enters the channel. Leakage direction is determined for each segment in communication with the channel, by comparing each segment-chamber pressure and channelend pressure. Leakage rates with the forward segment $\dot{m}_{A}$ and the rear segment $\dot{m}_{B}$ are obtained using loss curves described later, given the upstream static pressure and temperature, downstream pressure. channel geometrical characteristics such as width and height, and portion of the channel width opened to the segment. The described procedure is conducted for both inner and outer radii, at the left and right rotor ends. With subscripts $O$ and $l$ referring to outer and inner gaps, respectively, the total leakage flow for one end of the channel at each time step is the sum of flows for all communicating segment:

$$
\dot{m}(t)=\left[\dot{m}_{A}+\dot{m}_{B}\right]_{o}+\left[\dot{m}_{A}+\dot{m}_{B}\right]_{l}
$$

The amounts of leakage mass accumulated in sequential chamber $j$ ahead of the channel and $j-1$ behind the channel are

$$
\dot{m}_{j}=\sum_{\theta=\theta_{j}}^{\theta=\theta_{j+1}} \dot{m}_{A}\left(\frac{\Delta \theta}{\theta_{\text {cycle }}}\right), \quad \dot{m}_{j-1}=\sum_{\theta=\theta_{j}}^{\theta=\theta_{j+1}} \dot{m}_{B}\left(\frac{\Delta \theta}{\theta_{\text {cycle }}}\right)
$$

where $\theta_{j}, \theta_{j+1}, \Delta \theta$, and $\theta_{\text {cycle }}$ are the segment-start boundan. segment-end boundary, time step, and cycle period, respectivel: Note that when a single segment is encountered, $\dot{m}_{B}=0$ is set in th calculation.

At each leakage chamber, it is useful to calculate the averge temperature and pressure of the entering and leaving flows. Suxh averages are meaningful only if the flow direction is fixed; yet in possible that while the channel is traversing a given segment, leakage flow may reverse direction as waves in the channel change pressure. Therefore, the code calculates average pressure at temperarure separately for the positive flows (entering the chandi) and negative flows (leaving the channel) based on the drivit pressure difference.

In the original single-cavity Q1D code, the converged solution obtained when cavity properties equilibrate, total net leakage tends 
zero, and port flows reach steady state. With segment properties now fixed during each iteration of the Q1D calculation, only local leakage rates are calculated, and the accounting of mass balance is now accomplished within the flow network solver, described next. A revised Q1D local convergence criteria set is satisfied when port flows are steady and relative changes in each leakage mass flow rate from one cycle to the next become small compared with the current value for that segment; for example,

$$
\left|\frac{\dot{m}_{j}\left(t_{\text {cycle }+1}\right)-\dot{m}_{j}\left(t_{\text {cycle }}\right)}{\dot{m}_{j}\left(t_{\text {cycle }}\right)}\right| \leq \varepsilon \quad \text { for all } j
$$

where $j$ represents each leakage chamber, and $\varepsilon$ is a small value, typically 0.01 . It should be noted that individual leakage flow for any segment is a small fraction of the main port flows. Even for the largest segment-leakage rates, the preceding convergence criterion makes the precision in leakage prediction better than 0.0001 of the inlet mass flow. For model validation, the measurement of such small tows is a challenge and is currently of limited accuracy.

\section{Flow Network Solver}

Analysis of a complex network of leakage flows within a gasurbine engine is generally conducted using a model network of restrictor and chamber elements and a flow-solution method. These methods are generally proprietary to specific gas-turbine manufacturers. The favored modeling approach consists of determining key potential leak paths and a set of common regions with definable pressures. Connecting the regions are discrete flow restrictions with defined geometry and predictable pressure-loss characteristics. This work applies pressure-loss characteristics using the geometrically based $K$-factor loss method for commonly encountered passage geometries [18] or, alternatively, an empirical loss-flow curve based on test data, when necessitated by unique geometry.

The method was validated by long experience in gas-turbine secondary-flow modeling. In the resulting flow analysis network, unknown pressures and flows are solved using known boundaryvalue pressures and temperatures set typically by atmospheric or gasflowpath values. The strongest pressure gradients presented to lakage-prone regions are in the circumferential and radial directions; the network model used here was specifically designed to represent those leakages.

Such an analysis tool has now been applied to the wave rotor to solve for the leakage flows within the device as a whole, in conjunction with the main channel flows. The method closely integrates the leakage flow solver with the revised Q1D analysis tool described previously. Within gas-turbine design and analysis, basic leakage levels are generally established using estimates of main gaspath pressures and temperatures, from which leakage-flowpath solutions in the compressor or turbine are generated using applicable tools. Changes in the leakage levels do not strongly influence the gaspath flow solutions. In contrast, within the wave rotor, it was coserved that gas-path solutions in the channels can be affected significantly by leakage flows. Hence, a methodology was formulated that links and iteratively solves for the solution of both leakages and channel properties together. The methodology allows the leakage solutions of the computational fiuid dynamics model to onverge to levels common with the leakage network model, with murual feedback. The two models share a set of common interface lakage flow, pressure, and temperature values. The method thus the Q1D and network solver programs independently but acperatively to step through a series of iterations sharing leakage ifformation and the same gap-resistance mode. A solution is chieved when the exchanged pressures and temperatures each converge to a common value, as shown in the solution procedure Aowchan of Fig. 8.

Although leakage pathways in the various cavities of the casing ure relatively straightforward to model, special attention is paid to the ratial and circumferential pathways adjacent to the rotor and their riving pressure gradients. Regions of similar pressure around the

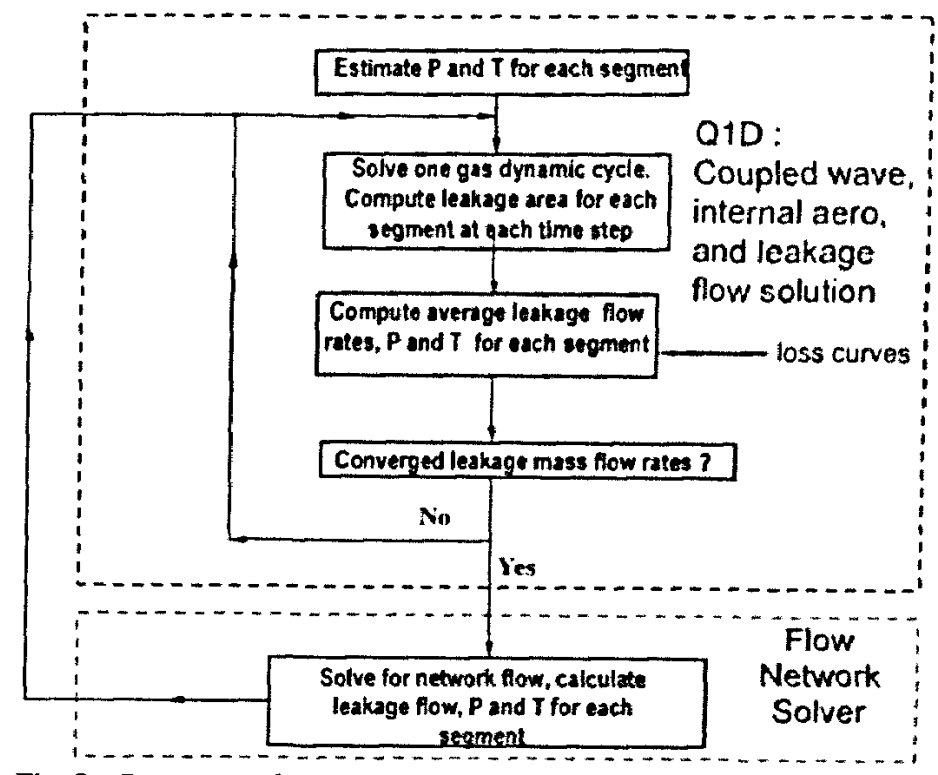

Fig. 8 Summary of solution strategy for leakage calculation by QID and the flow network solver.

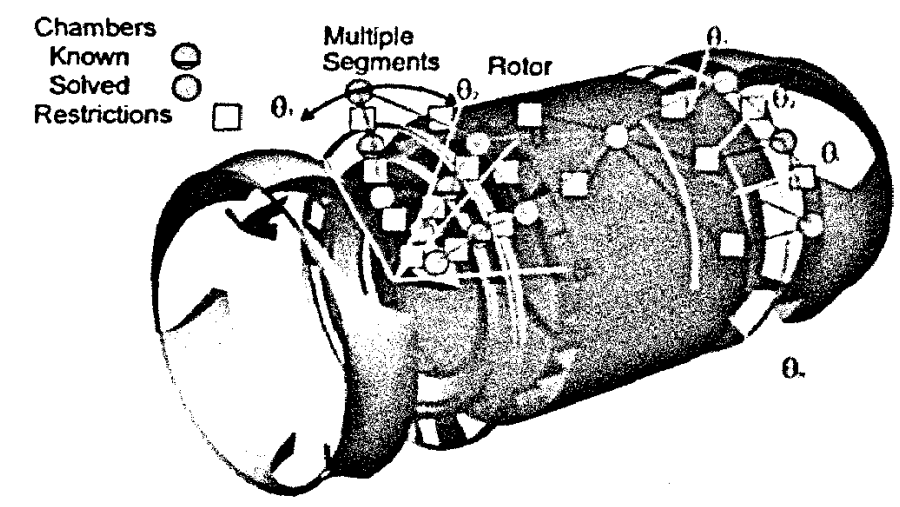

Fig. 9 Schematic of leakage network solver methodology for generalized wave-rotor configuration.

circumference are identified and treated as distinct leakage regions. Part of a typical set of chamber elements configured around a rotor is schematically represented in Fig. 9, illustrating detailed representation of rotor-to-end-plate interface regions and additional network elements connecting the two interface planes. In typical wave-rotor geometry, the intersegment resistances (around the circumference) may be comparable with the resistances within the segments. Thus, intersegment resistances are estimated to create a discrete representation of the distributed resistance to the circumferential pressure field. In most designs. this fow is not a dominant feature and can be well accounted by the simplified resistance model. More important, the segmentation allows zones of widely varying temperatures and gas density to be differentiated. This allows better prediction of thermal effects and leakage mass flows and allows the design of seal geometries to impose a higher degree of flow restriction, as needed to sustain the pressure field or confine the temperature field. This feature of the method developed here has significantly improved wave-rotor design methods with respect to managing the effects of leakage.

\section{v. Simultaneous Solution of Wave Solution and Leakage-Flow Network}

In the solution strategy, the QID and flow network solvers are used in a sequential and iterative manner. The QID wave-rotor flow solver is first used to predict the leakages based on an initial guess of the pressures within the leakage chambers adjacent to the rotor-endplate gap. It achieves a converged solution of the transient rotor 
Table 1 Leakage-modeling results compared with static-rotor tests on a wave-rotor rig

\begin{tabular}{cccc}
\hline \hline Test run no. & Predicted mass flow rate, $\mathrm{kg} / \mathrm{s}$ & Measured mass tlow rate, hg/s & Differtince, r \\
\hline 3417 & 0.0143 & 0.0150 & 5 \\
3423 & 0.0115 & 0.0118 & 3 \\
3426 & 0.0129 & 0.0150 & 16 \\
\hline
\end{tabular}

channel and leakage-gap flow rates. The leakage-flow rate to each segment chamber represents a space integral over all channels in communication with it and a time-integral over the channel-opening/ closing time. The predicted wave-rotor-segment leakage flows, either into or out of the leakage chambers, are then used by the flow network solver to determine a converged leakage network solution. This is based on a balance of flow and pressure loss in the overall leakage-flow network external to the rotor channel, which may also include a net flow either entering or leaving the system. A revised set of leakage-chamber pressures is thus created. The QID solver then creates a new rotor-channel solution using these new leakagechamber pressures. This results in revised leakage-flow rates. An overall converged solution is achieved when successive applications of the Q1D and flow network solvers arrive at predictions of leakage within a defined convergence tolerance based on changes in absolute magnitude of leakage flow for each circumferential segment.

The network solver employed had been designed to use known boundary values in terms of known pressures, not known leakages. Thus at the boundary to the wave-rotor channel, a set of channel pressures is determined for each segment using the Q1D-predicted gap leakage flows, the leakage-chamber pressures used by Q1D in solving for this leakage flow, and the pressure-drop characteristic of the gap leak path. This set of contrived pressures is then used as the known boundary pressures to arrive at a converged flow network solution. The network solver then carries out a subiteration to arrive at new rotor-channel and leakage-chamber pressures that match the given flow rates. Thus, the leakage gap represents an overlapping region of the two solver domains, and both solvers use a common flow versus pressure drop characteristic of the gap leak path at the rotor-to-chamber interface to ensure consistency. It should be noted that the temperature of the leakage flow is determined by the QID solution when the net leakage is from the rotor channel into a leakage chamber. Conversely, the temperature of the leakage flow is determined by the flow network solver when the flow is from the leakage chamber into the wave-rotor gas flowpath.

Although the two codes are called sequentially in this work, they are coupled well by the overlapped treatment of the primary leakage gap. Envisioned in future design practice are a closer coupling of the Q1D tool and the flow network solver, improved identification of appropriate segments, and loss estimation, and modeling of direct leakage between channels of the rotor. The current approach takes $n$ important step in methods development for analysis and design of wave rotors.

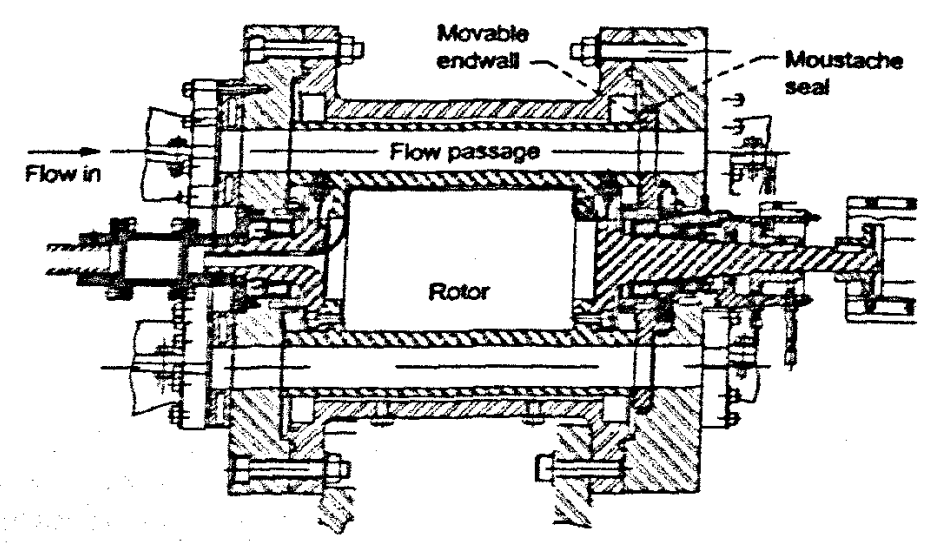

Fig. 10 Cross-sectional view of the assembled wave-rotor rig, from [19]

\section{Application to Wave-Rotor-Rig Leakage Evaluation}

The described methodology was applied to the analysis of leakage in an experimental pressure-exchange wave rotor, shown in Fig. 10 [19], intended to test the feasibility of wave-rotor topping for gasturbine performance enhancement [20] using the wave-rotor topping cycle of Fig. 2. Wave-rotor development is at an early stage, in which basic physics and losses associated with the nonsteady flow are reasonably well understood. but important mechanical design issues are being addressed, including those that control leakage. The operating cycle of this particular wave rotor involves four ports: high-pressure and low-pressure inlets on one side and high-pressure and low-pressure outlets on the opposite side.

A model of the entire internal leakage network was generated using detailed geometric information to create a network flow circuit and pressure-versus-flow characteristics for each leakage-flowcircuit resistance element. Geometric information was gleaned from rig drawings, build-clearance measurements, and visual inspection. To valicate the steady-state-flow network-model representation, the network model was first exercised for a nonrotating mode with staticrotor flow conditions, corresponding to experimental runs in this mode. For the static-rotor case, the channel-to-channel leakage paths were determined to be important and were included in the network model for this case. No Q1D results were needed in this case because no transient flow was present within the rotor channels. As Table 1 shows, results from several static-rotor tests on the four-port wave rotor were used to validate the detailed leakage-circuit model. Despite uncertainties in leakage-flow measurement employinglargeorifice plates to measure small flow rates, due to Reynolds number effects on loss coefficients, the agreement between the test and predicted results was encouraging.

Transient operation of the experimental wave rotor was then considered. A Q1D simulation of one rig test point, run 3468 , was performed using the single-cavity version of $Q 1 D$. The value of the single-cavity pressure and temperature was then used as the initial value for each of the leakage-chamber pressures and temperatures. Guided by port locations and by the initial simulation results for circumferential pressure and temperature distribution, as showi in Fig. 11, the (left) inlet end of the rotor at the rotor-end-plate gap was divided into five segments circumferentially, and the (right) exit end was divided into seven segments. The selection of segments must necessarily be made based on hardware geometry and preliminary gas-dynamic predictions of rotor pressures. Because pressure determines leakage direction, resolution of pressure variation is given primary consideration above resolution of segment boundaries with respect to temperature variations. Use of a greater number of segments might predict leakage flows more accurately, but with additional computing and intercode communication effor, if justified. in design methodology. The preceding selection was not altered or improved during this study.

Based on the chamber initial values and on the space-averaged wave-rotor-channel end-plate properties, the flow network solver was used to predict a leakage network solution (iteration 0). The predicted leakage-chamber values were then used by the $Q L D$ program to solve for a new wave solution within the rotor and new leakage values (iteration 1). The solution history of QID and the flow-network-solver inlet and exit nomalized leakage flows are shown in Figs. 12 and 13. The predicted pressure and leakage-fiow values were nomalized by the run 3468 experimentally meastred total pressure and mass flow rate levels corresponding to intit station 1 (see Fig. 2). Convergence was judged to be achieved whan 

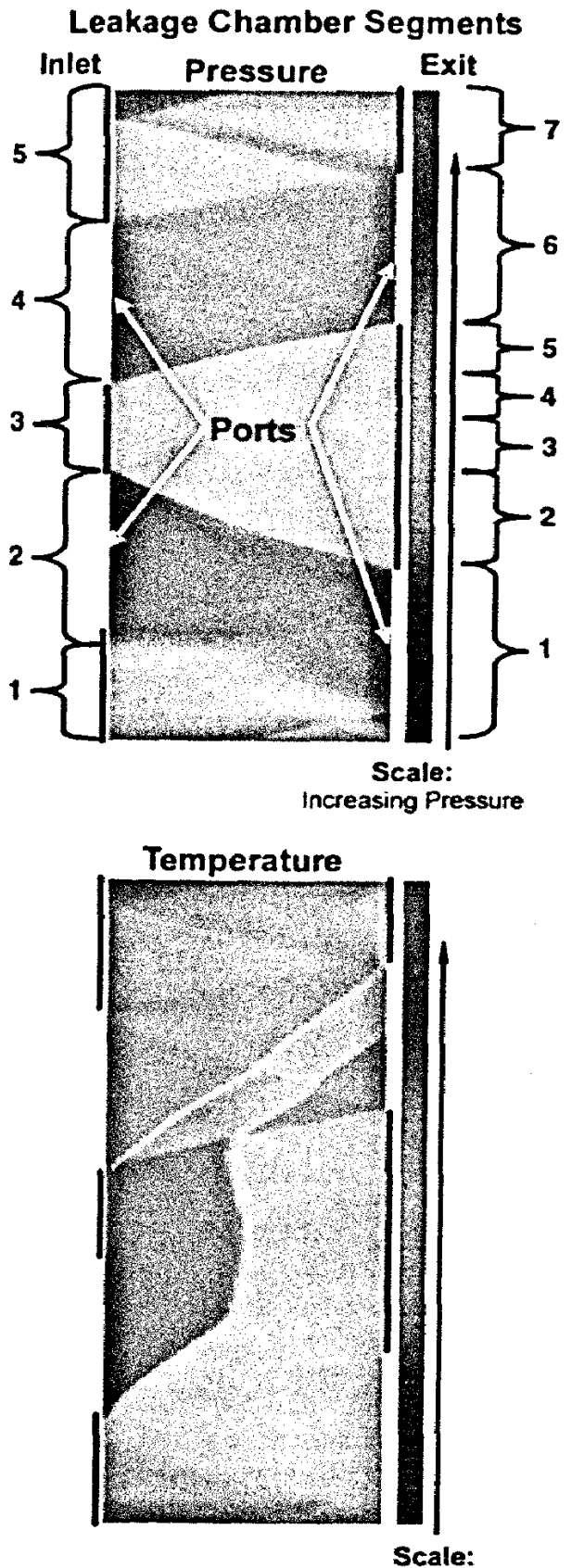

Increasing Temperature

Fig. 11 Developed plot of rotor pressure and temperature predicted by Q1D with leakage-chamber segments and ports shown.

the magnitude of change in QID-calculated leakage flows from iteration to iteration fell below $0.000023 \mathrm{~kg} / \mathrm{s}$. In comparison, the overall inlet mass flow rate (station 1 in Fig. 2) of the device is approximately $0.34 \mathrm{~kg} / \mathrm{s}$, with a station 1 total pressure of $52 \mathrm{kPa}$ and total temperature of $226 \mathrm{~K}$. It may be noted that the leakage rates are generally under $1 \%$ of the primary inlet mass flow rate. and the leakage-segment pressures stabilize at levels intermediate between the high and low pressures of the cycle. It is also interesting that for some segments, there is a significant difference between inner- and outer-segment pressures, due to the different network topology and resistances. After iteration 0, the leakage values changed very little and convergence was quickly achieved. In each of these global iterations, the flow network solver typically needed less than 100 subiterations, and the Q1D code typically needed about 10 periodic gas-dynamic cycles. As shown in Figs. 14 and 15, global convergence of pressure was achieved very rapidly, within six global iterations for the case presented.

To assess the potential for improvement in leakage control, it was instructive to compare the resulting converged flow and pressure solutions for the rig experimental point with two additional test cases

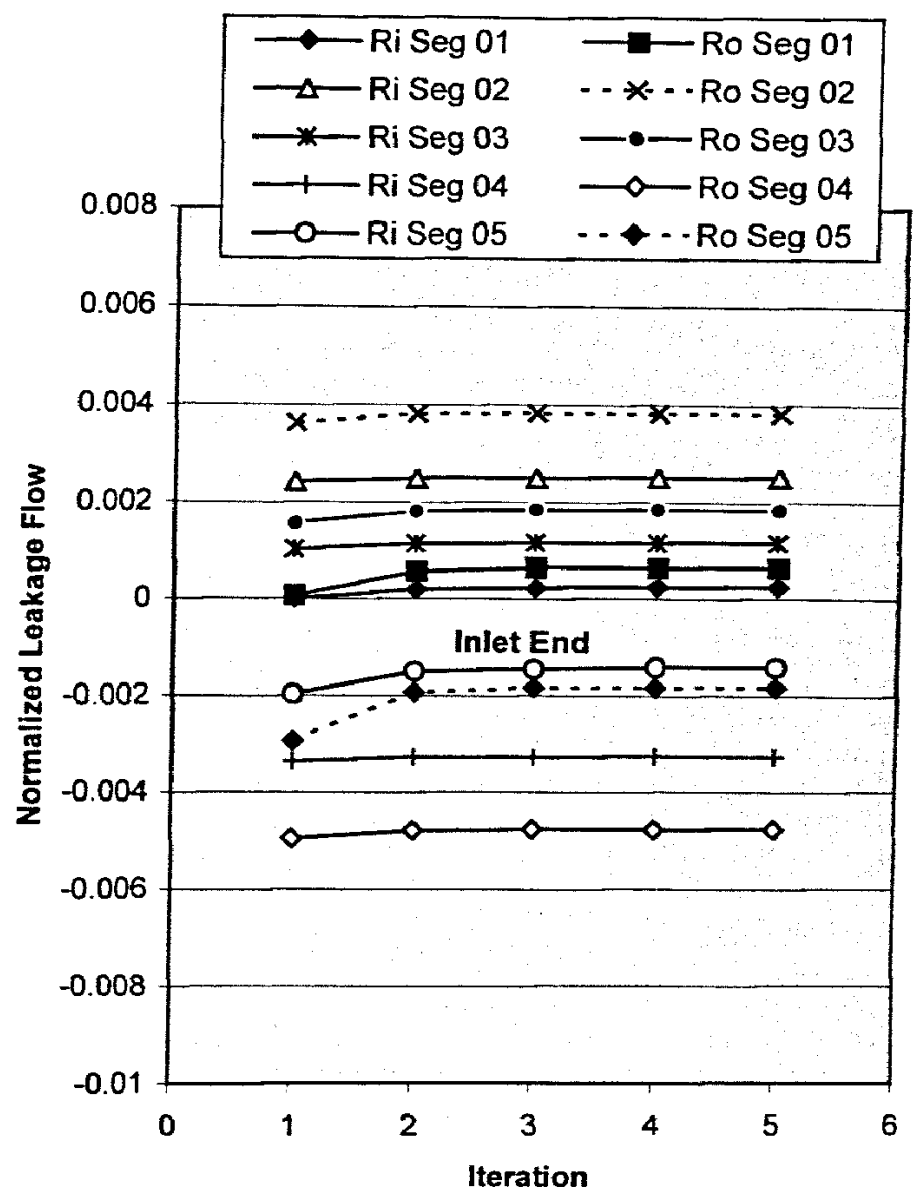

Fig. 12 Solution history for the inlet-end rotor-segment leakage flow, run 3468.

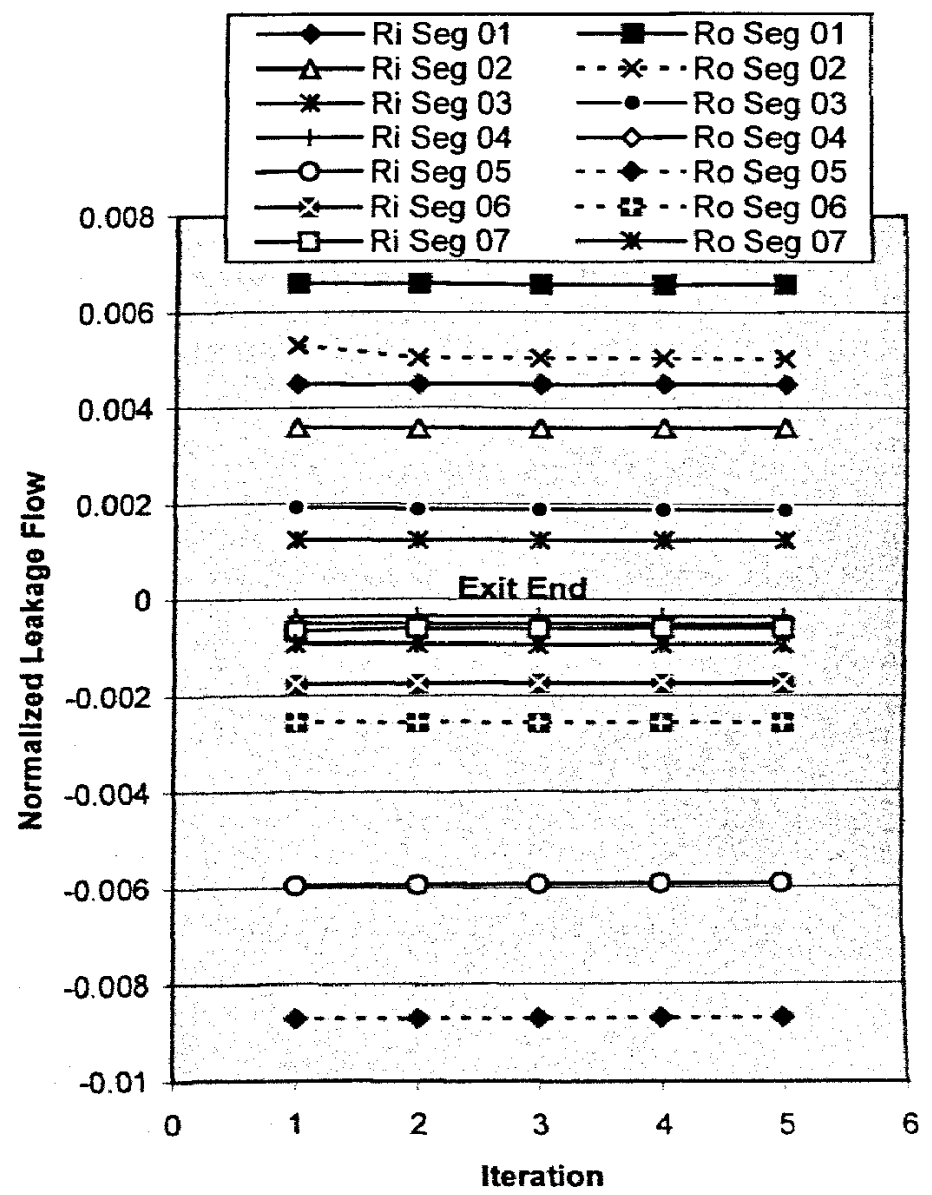

Fig. 13 Solution history for the exit-end rotor-segment leakage flow, run 3468 . 


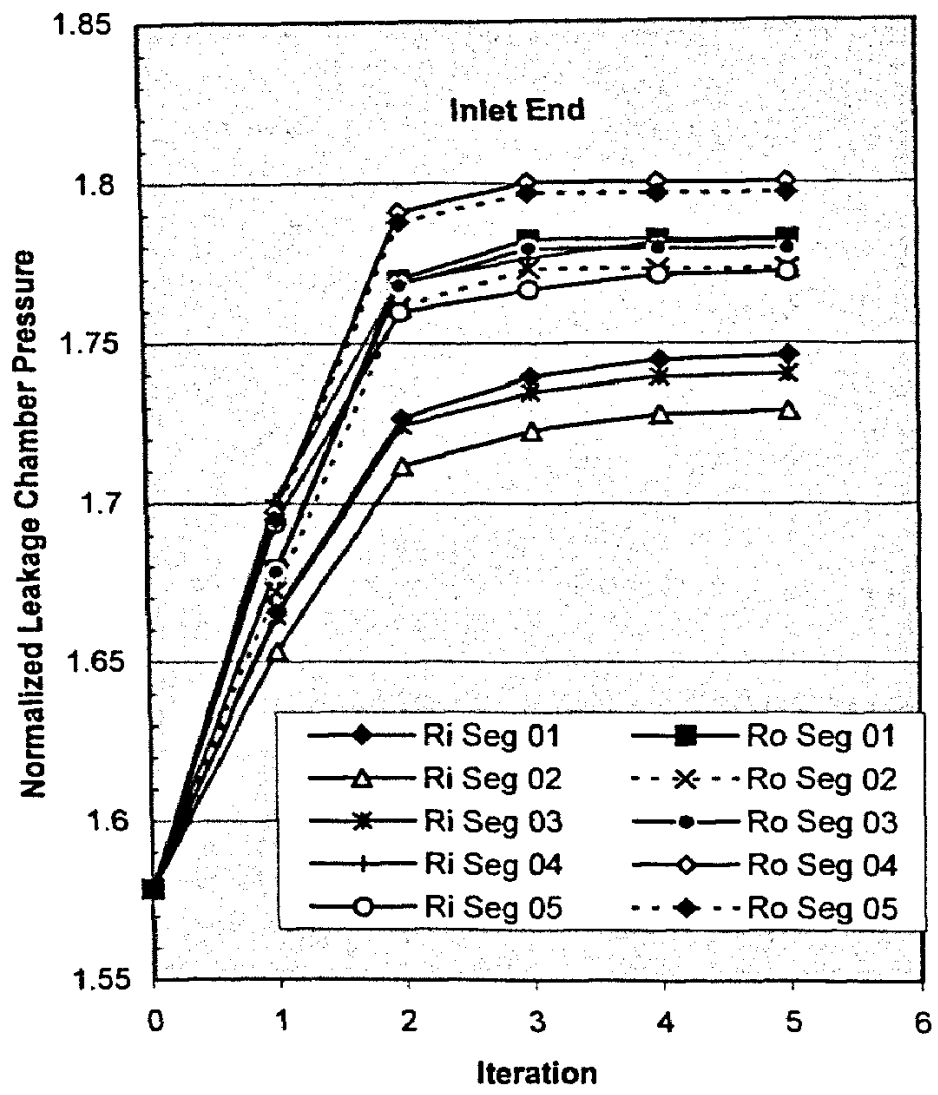

Fig. 14 Solution history for the inlet-end leakage-chamber pressures, run 3468.

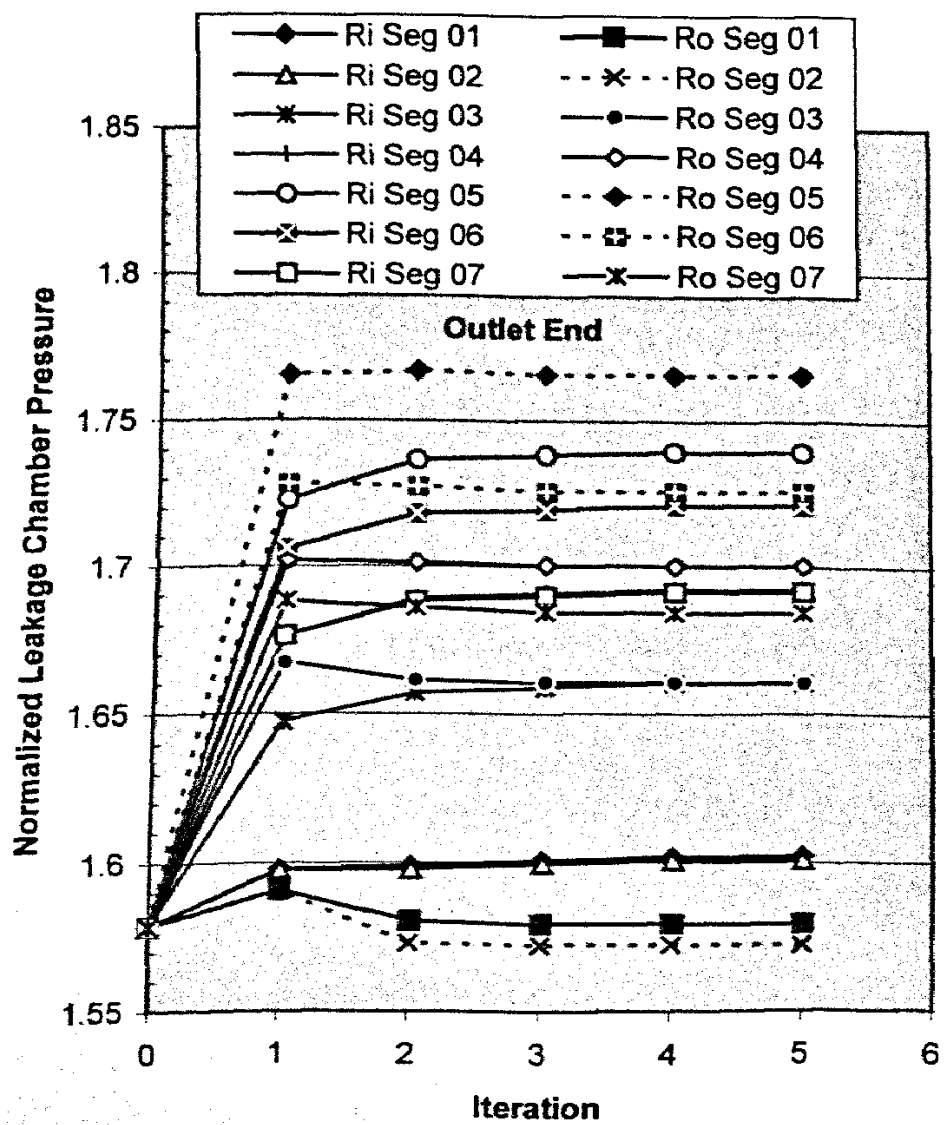

Fig 15 Solution history for the exit-end leakage-chamber pressures, $\operatorname{ran} 3468$.

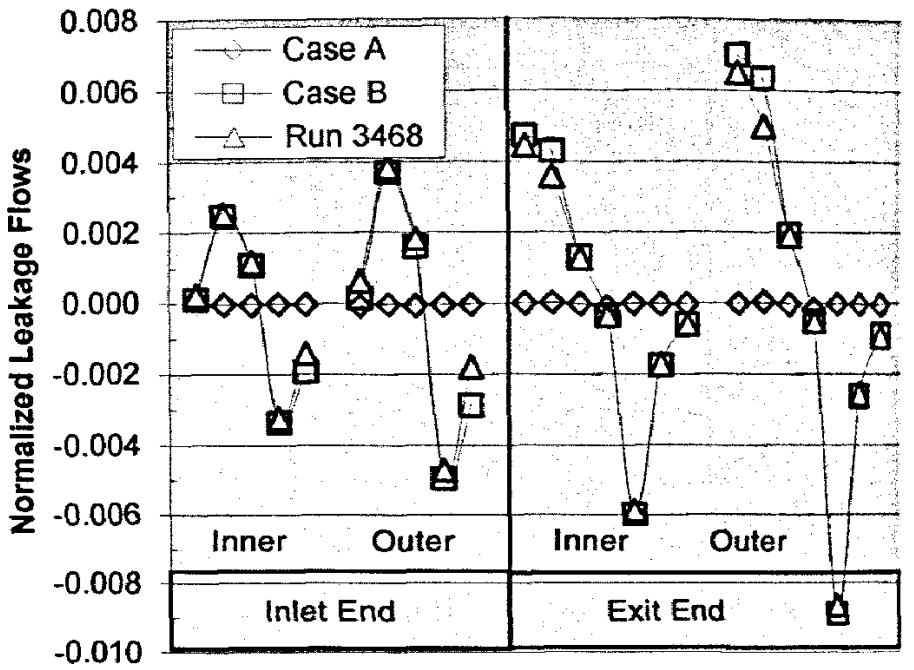

Segment

Fig. 16 Comparison of rotor-channel leakage flows.

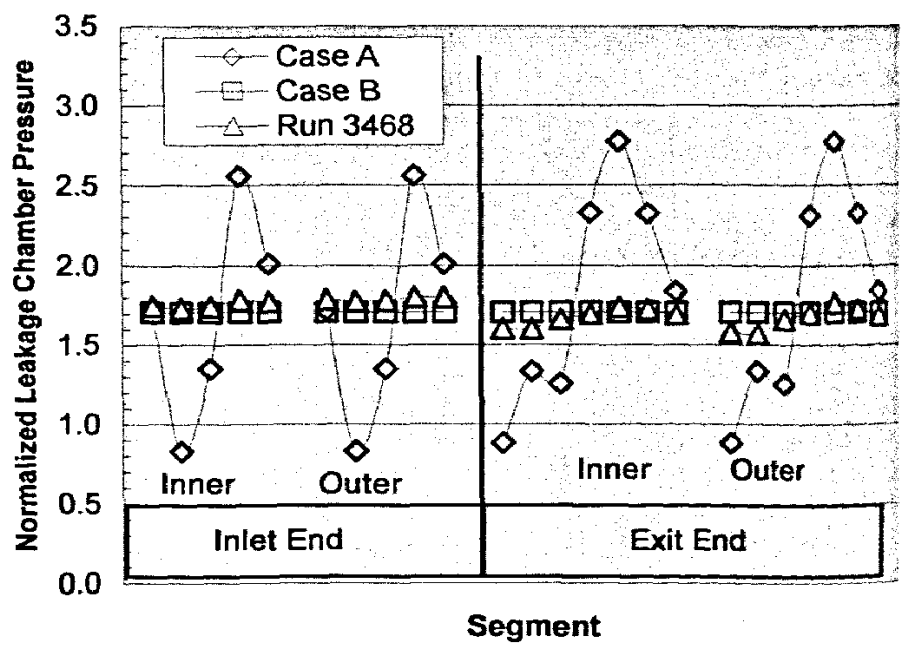

Fig. 17 Comparison of rotor leakage-chamber pressures.

that examine extremes of the network-model solution domain. In both cases, the flow resistance between the rotor channels and the leakage chambers was maintained at a baseline geometry reflecting established baseline gap dimensions on the inlet end and outlet end of the rotor.

Case A examined an external leakage circuitry in which circumferential sectors of the solution leakage chambers were completely isolated from the remainder of the leakage network. The resistances between these leakage chambers and all regions other than the rotor channel via the endwall gap were set to artificially high levels. With no communication allowed between circumferentis segments and any other part of the leakage circuit, leakage exists only between the rotor channels and the leakage chambers within a segment. As anticipated, the converged solution resulted in leakgechamber pressures closely matching average rotor-channel gasflowpath pressure levels for each of the respective segments.

Conversely, case B allowed perfectly unrestricted communication in each of the identified leakage paths external to the rotor-endwil gap, but retained baseline rotor-end-plate leakage-gap chararistics. Thus, the leakage chambers (left and right and inner and out radii) all reached a common pressure prescribed by an extema boundary condition determined from test data. Run 3468 resuls reflected experimental-test hardware-imposed conditions. Each of the test cases and the experiment-matching case considered identics rotor-endwall gap levels as deduced from cold build data adjusta for rotor thermal expansion.

Figures 16 and 17 present comparisons of normalized fow leakage and nomalized chamber pressure, respectively. It was 
Table 2 Summary of leakages, run 3468

\begin{tabular}{lcccc}
\hline \hline $\mathrm{kg} / \mathrm{s}$ & \multicolumn{2}{c}{ Inlet } & \multicolumn{2}{c}{ Exit } \\
\hline & Entering & Leaving & Entering & Leaving \\
\hline Inner radius & 0.00148 & 0.00125 & 0.00209 & 0.00200 \\
Outer radius & 0.00209 & 0.00200 & 0.00273 & 0.00297 \\
Total & 0.00357 & 0.00325 & 0.00677 & 0.00724 \\
Entering flow minus leaving flow & \multicolumn{2}{c}{0.00032} & & -0.00047 \\
\hline \hline
\end{tabular}

Table 3 Pressure-gain results of three cases

\begin{tabular}{lcc}
\hline & $P_{4} / P_{1}$ & $T_{4} / T_{1}$ \\
\hline Case A & 1.143 & 2.013 \\
Case B & 1.116 & 1.994 \\
Run 3468 & 1.116 & 1.992 \\
\hline \hline
\end{tabular}

expected that the distribution of leakages and pressures for the run 3468 case would be bracketed by that for case A and case B. Although this was indeed the trend, leakage rates and leakagechamber pressures for run 3468 were very close to those for case B, the perfect communication case. This indicated that leakage flow for nun 3468 was not highly restricted, either from one end of the rotor to the other, or in the circumferential direction. Leakage distribution around the rotor circumference showed the consequent outflow from the rotor channels at the high-pressure side to the low-pressure side at each end. Table 2 reports the net outflow and inflow components.

The results also indicate a net casing flow of $0.00032 \mathrm{~kg} / \mathrm{s}$ axially from the inlet end to the exit end. This agreed with the difference between the entering and leaving flows on the inlet end of the rotor as predicted by Q1D. The unequal value of the difference between the entering and leaving flows on the inlet and exit ends of the rotor was due to a significant and surprising leakage flow on the exit end to a low-pressure exit-port region through a path independent of the rotor-end-plate gap. The identification of this pathway in this study, and later experimental mitigation of this leakage, was a significant step in recent wave-rotor technology.

The effects of changes in leakage flow on overall performance of the wave rotor as predicted by the Q1D code, including detailed leakage modeling, are listed in Table 3 . The station numbering convention is that of Fig. 2 . It is significant to note that an additional pressure gain $P_{4} / P_{1}$ of $3 \%$ is predicted for case $A$, in which the leakage flow is constrained within defined circumferential sections of similar rotor-flowpath pressure. This represents the relative potential enhancement in wave-rotor performance available from the use of improved sealing features between chambers, including the rotor-endwall clearance gap.

Following the analytical work described, modifications were made to the wave-rotor rig reflecting certain lessons learned regarding leakage behavior. This included a reduction of leakage by a circumferential path separate from the rotor clearance gap, corresponding to the "moustache seal" behind the moveable endwall, shown in Fig. 10. Subsequent testing of the wave-rotor rig reported a significantly improved pressure gain, comparable with the pressure gain of case A [19]. This reported performance is consistent with improved leakage control of pathways other than the clearance gaps. Detailed geometric information for the improved design were not arailable for quantitative comparison. Nevertheless, it tends to confirm the indication from this study that wave-rotor leakage control requires all paths between high- and low-pressure regions to be carefully evaluated and designed.

\section{Conclusions}

The methodology developed in this work combined the Q1D wave-rotor unsteady-flow code with a network flow solver to predict teakages within a wave-rotor system with significantly improved fdelity to circumferential variation in leakage rates and pressures and to casing fows beyond the rotor-stator gap. Design of the wave-rotor casing-cavity flowpaths external to the wave-rotor channel and clearance gap can significantly influence performance due to overall leakage effects, which are now included in the gas-flowpath dynamics. Sealing techniques and other leakage-defeating measures can be assessed for influence on wave-rotor performance. Pressures and temperatures of the leakage gas flows surrounding the rotor can be predicted more accurately.

The insights provided regarding the details of the wave-rotor leakage flows and their effects can lead to the development of improved methods to minimize leakages and enhance wave-rotor performance. Data from an experimental pressure-exchange waverotor rig were examined to construct a leakage network model. Improved performance obtained after addressing leakage paths in the rig identified and modeled in this work was consistent with the trends predicted. The segmented modeling of leakage according to regions with different temperatures will also improve the thermal-protection features that address heat transfer due to leakage flows. This capability can then lead to improved designs of wave-rotor components. Ultimately, successful wave-rotor design will enable gas-turbine performance levels beyond those achievable through evolutionary turbomachinery component improvements.

\section{Acknowledgments}

The authors would like to gratefully acknowledge D. E. Paxson at NASA John H. Glenn Research Center at Lewis Field for his helpful advice and comments. This work was funded from the NASA Low Emissions Alternative Power, Constant-Volume Combustion Cycle Engine (LEAP CVCCE) project (Leo Burkardt, Element Manager, and Gerard Welch, Contract Technical Manager) as part of NASA contract NAS3-01143, task order 5, Seal Technology Development for Advanced Components for Airbreathing Engines.

\section{References}

[1] Seippel, C., Swiss Patent 225426, 1940.

[2] Berchtold, M., "The Comprex," ONR/NAVAIR Wave Rotor Research and Technology Workshop, Naval Postgraduate School, Rept. NPS -6785-008, Monterey, CA, 1985. pp. 50-74.

[3] Rose, P. H., "Potential Applications of Wave Machinery to Energy and Chernical Processes." Proceedings of the 12ih International Symposium on Shock Tubes and Waves, Magnes Press, Jerusalem. 1979, pp. 3-30.

[4] Wilson, J., "An Experimental Determination of Loses in a Three-Pon Wave Rotor," Journal of Engineering for Gas Turbines and Power, Vol. 120, No. 4, 1998. pp. 833-842. doi: $10.1115 / 1.2818476$

[5] Kharazi, A. A., Akbari, P., and Müller, N., "Preliminary Study of a Novel R718 Compression Refrigeration Cycle Using a 3-Port Condensing Wave Rotor," Joumal of Engineering for Gas Turbines and Power, Vol. 127, No. 3, 2005, pp. 539-544. doi: $10.1115 / 1.1850503$

[6] Fatsis, A., and Ribaud, Y., "Thermodynamic Analysis of Gas Turbines Topped with Wave Rotors," Aerospace Science and Technology, Vol. 3, No. 5, 1999. pp. 293-299. doi: $10.1016 / S 1270.9638(00) 86965-5$

[7] Nalim, M. R., "Thennodynamic Limits of Work and Pressure Gain in Combustion and Evaporation Processes," Joumal of Propulsion and Power, Vol. 18, No. 6, 2002, pp. 1176-1182.

[8] Shreeve, R. P., and Mathur, A.. ONRNAVAIR Wave Rotor Research and Technology Workshop, Naval Postgraduate School, Rept. NPS-6785-008, Monterey, CA, 1985.

[9] Akbari, P., Nalim, M. R., and Müller, N., "A Review of Wave Rotor Technology and Its Applications," Joumal of Engineering for Gas Turbines and Power, Vol. 128, No. 4, 2006, pp. 717-735. doi: $10.1115 / 1.2204628$ 
[10] Akbari, P., Müller, N., and Nalim, M. R., "Performance Enhancement of Microturbine Engines Topped with Wave Rotors," Journal of Engineering for Gas Turbines and Power, Vol. 128, No. 1, 2006, pp. 190-202.

doi: $10.1115 / 1.1924484$

[11] Kentfield, J. A. C., "On the Feasibility of Gas-Turbine Pressure-Gain Combustors," International Journal of Turbo and Jet Engines, Vol. 12, No. 1, 1995, pp. 29-36.

[12] Roy, G. D., Frolov, S. M., Borisov, A. A., and Netzar, D. W., "Pulse Detonation Propulsion: Challenges, Current Status, and Future Perspective," Progress in Energy and Combustion Science, Vol. 30, No. 6, 2004, pp. 545-672.

doi:10.1016/j.pecs.2004.05.001

[13] Akbari. P., Szpynda E., and Nalim, M. R., "Recent Developments in Wave Rotor Combustion Technology and Future Perspectives: A Progress Review," AIAA Paper 2007-5055, 2007.

[14] Paxson, D. E., "Comparison Between Numerically Modeled and Experimentally Measured Wave-Rotor Loss Mechanisms," Journal of Propulsion and Power, Vol. 11, No. 5, 1995, pp. 908-914.

[15] Hendricks, R. C., Wilson, J., Wu, T., and Flower, R., "Two-Way Brush Seals Catch a Wave," Mechanical Engineering, Vol. 120, No. 11, 1998, pp. 78-80.

[16] Paxson, D. E., "Numerical Simulation of Dynamic Wave Rotor Performance," Journal of Propulsion and Power, Vol. 12, No. 5, 1996, pp. 949-957.

[17] Nalim, M. R., and Paxson, D. E., "A Numerical Investigation of Premixed Combustion in Wave Rotors," Joumal of Engineering for Gas Turbines and Power, Vol. 119, No. 3, 1997, pp. 668-675. doi:10.1115/1.2817036

[18] Miller, D. S., Internal Flow Systems, Vol. 5 BHRA Fluid Engineering, Cranfield, England, U.K., 1978.

[19] Wilson, J., Welch, G. E., and Paxson, D. E., "Experimental Results of Performance Tests on a Four-Port Wave Rotor," AIAA Paper 2007. $1250,2007$.

[20] Welch, G. E., Jones, S. M., and Paxson, D. E., "Wave Rotor-Enhanced Gas Turbine Engines," Journal of Engineering for Gas Turbines and Power, Vol. 119, No. 2, 1997, pp. 469-477.

doi: $10.1115 / 1.2815598$

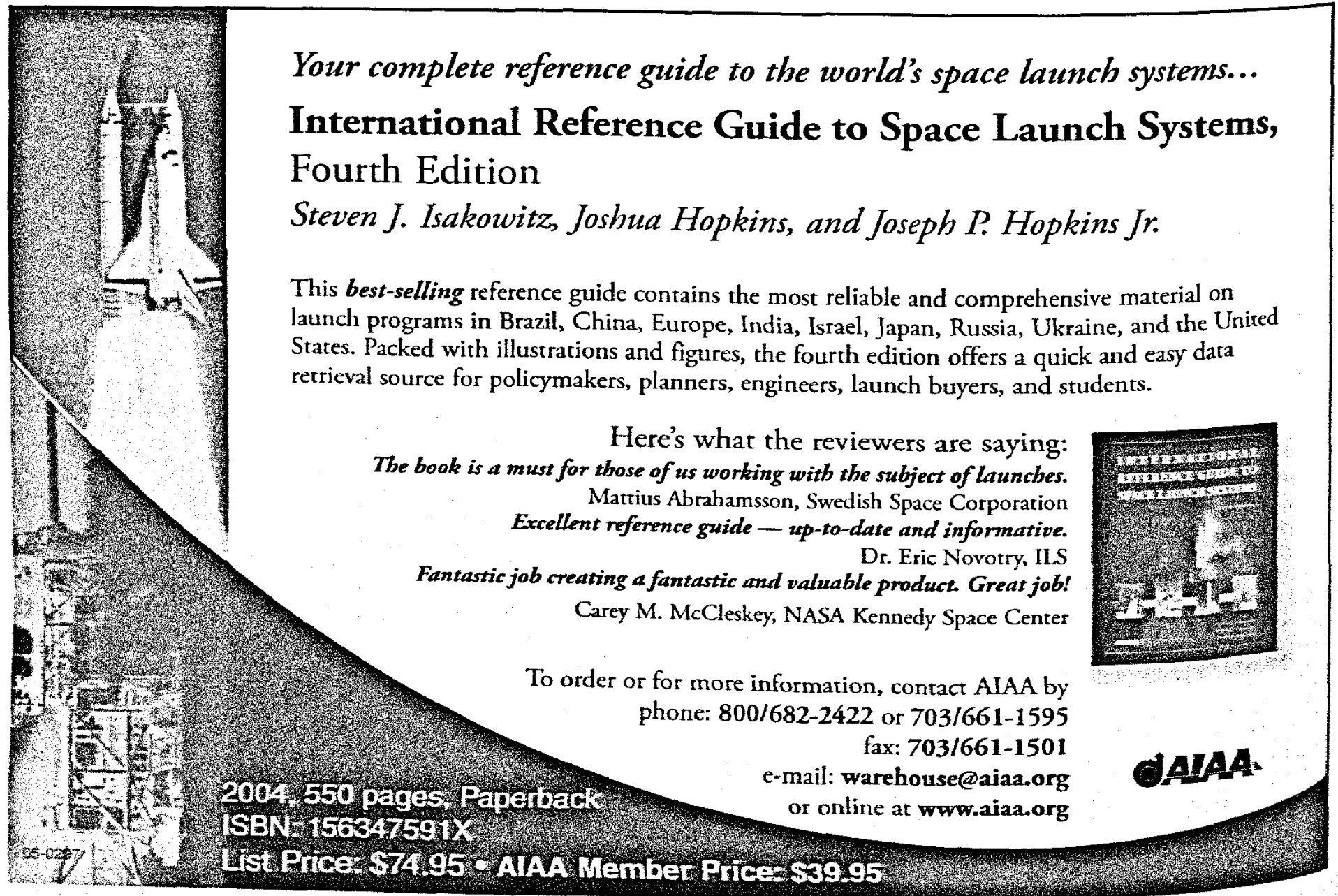

